Voix et Images

voixetimages

\title{
Parlez-vous littérature?
}

\section{Sylvain Simard}

Volume 18, numéro 1 (52), automne 1992

Les écritures masculines

URI : https://id.erudit.org/iderudit/201006ar

DOI : https://doi.org/10.7202/201006ar

Aller au sommaire du numéro

Éditeur(s)

Université du Québec à Montréal

ISSN

0318-9201 (imprimé)

1705-933X (numérique)

Découvrir la revue

Citer cet article

Simard, S. (1992). Parlez-vous littérature? Voix et Images, 18(1), 152-156.

https://doi.org/10.7202/201006ar

Ce document est protégé par la loi sur le droit d'auteur. L'utilisation des services d'Érudit (y compris la reproduction) est assujettie à sa politique d'utilisation que vous pouvez consulter en ligne.

https://apropos.erudit.org/fr/usagers/politique-dutilisation/
Cet article est diffusé et préservé par Érudit.

Érudit est un consortium interuniversitaire sans but lucratif composé de l’Université de Montréal, l'Université Laval et l'Université du Québec à Montréal. Il a pour mission la promotion et la valorisation de la recherche. https://www.erudit.org/fr/ 
1. Jean Marcel, Pensées, passions et proses, Montréal, L'Hexagone, coll. -Essais littéraires , 1992, $399 \mathrm{p}$.

2. Laurent Mailhot, Ouvrir le liere, Montréal, L'Hexagone, coll. eEssais littéraires", 1992, 353 p.

\section{Parlez-vous littératuré?}

\section{Sylvain Simard, Université d'Ottawa}

«[Tout] est accroché à cet élément essentiel, en découle ou nous y ramène infailliblement. (René Lévesque, Option Québec, p. 19). Cet "élément essentiel", c'est, bien sûr, la langue; et dans "tout ce qui nous y ramène ", il y a la littérature, et ce depuis sa naissance au XIX ${ }^{c}$ siècle. Aussi, pour mieux connaître celle-ci, importe-t-il d'étudier non seulement l'état de la langue, mais aussi l'évolution des perceptions qu'en ont eues écrivains et critiques au Québec et en France.

L'auteure de Langue et Littérature au Québec, 1895-1914¹ appartient à cette équipe du Centre de recherche en littérature québécoise (CRELIQ) de l'Université Laval dont les nombreux travaux de qualité font beaucoup pour l'avancement de la connaissance sur la littérature d'ici. Cette spécialiste de l'œuvre critique de Charles ab der Halden privilégie une approche sociocritique inspirée surtout des travaux théoriques de Pierre Bourdieu et de ses collaborateurs des Actes de la recherche en sciences sociales. Inversant les termes habituels des rapports entre la langue et la littérature, Marie-Andrée Beaudet, dans cet essai fouillé, brillant et limpide, expose l'influence de la situation linguistique sur la formation d'un champ littéraire au Québec.

C'est d'ailleurs cette notion de "champ littéraire", plutôt que celle d'* institution littéraire, notion moins dynamique puisqu'elle implique une antériorité de la constitution et un certain déterminisme sur les "constituants", qu'adopte d'emblée l'auteur. Après avoir résumé de façon succinte le cadre linguistique général prévalant au Canada français, elle s'approprie, pour y donner suite, certaines hypothèses d'André Belleau sur le romancier québécois qui vit sa culture * dans la distance, comme le fait d'une autre classe, d'un autre pays [...]. et pour qui la situation linguistique concrète de l'écrivain québécois * nous oblige à poser le problème de la norme même de la langue littéraire [...]* à titre 
de condition institutionnelle indispensable s pour que le langage d'une communauté culturelle puisse recevoir et porter les codes de la littérature" (André Belleau, Le Romancier fictif, p. 176).

-Je suis un ignorant, je ne sais pas ma langue,, affirmait très modestement Albert Lozeau; déjà, les premières œuvres avaient donné lieu à des querelles linguistiques, et de Michel Bibaud à Fernand Ouellette, pratiquement toutes les œuvres littéraires posent, directement ou par critique interposée, le problème concret de la relation de l'écrivain à la langue. Notre histoire littéraire est d'abord l'histoire de nos débats linguistiques qui à leur tour posent le problème de la définition que notre société se donne d'elle-même et de ses rapports avec la France. Un discours valorise et pose même comme absolue la qualité certaine d'une langue qui serait celle de Racine ou de Bossuet maintenue miraculeusement intacte chez tous les parlant français d'Amérique ( $c f$. Sylva Clapin)! Ce discours est d'ailleurs encouragé par un certain nombre de critiques français aveuglés par leur amour des Canadiens. D'autres plus lucides, tels Alexis de Tocqueville ou $R$. de Marmande, mettront en évidence la dégradation de la langue parlée et écrite au Canada. Les Canadiens consacreront d'ailleurs des centaines de livres, opuscules et articles à dénoncer les faiblessés et à tenter de corriger la langue de leurs compatriotes. Les écrivains, souvent euxmêmes auteurs de ces écrits linguistiques, regrettent presque tous l'état de pauvreté de la langue parlée et écrite au Canada et surtout se plaignent de la très grande difficulté d'être lus et appréciés en France à leur juste valeur. Les Fabre, Fréchette et Beaugrand rejoignent ici leurs adversaires ultramontains et la virulence des dénonciations d'Arthur Buies n'est dépassée que par celle de Tardivel. L'objectif étant d'être lus, d'attirer l'intérêt de la critique et des lecteurs français, les écrivains ont presque tous conscience du handicap énorme que constitue une langue souvent déficiente. Certes Fréchette réussit un certain temps à attirer l'attention de la critique mais les illusions ne sont pas permises à ce sujet. Les témoignages nombreux établissent qu'il s'agit beaucoup plus d'une manifestation de sympathie à l'égard d'un représentant de ce peuple resté français en Amérique que d'une véritable reconnaissance de son talent d'écrivain.

Marie-Andrée Beaudet concentre sa démonstration sur les fondements linguistiques de la création d'une littérature nationale en étudiant deux dossiers: la querelle Jules Fournier et Charles ab der Halden et celle des exotistes et des régionalistes avec, au centre; la figure tutélaire de l'abbé Camille Roy. Ces dossiers permettent une excellente mise en lumière des enjeux du débat linguistico-littéraire dont ces affrontements ont été des abcès de fixation. 
La provocante affirmation de Fournier selon qui sll n'y a pas de littérature canadienne-française. La chose ne se discute pas." et les réponses du critique français sont ici l'objet d'une mise en situation quasi exhaustive et d'une analyse en profondeur. Les quatre lettres et un billet disparu qui occupent à peine trente pages dans la Revue canadienne sont d'abord présentés pour ce qu'ils sont, des illustrations fort concrètes de l'association langue et littérature. Si les lecteurs connaissent assez bien l'œuvre et la vie de Fournier dont on n'a évidemment rien dit lorsqu'on les a qualifiées de paradoxales et de contradictoires, le personnage d'ab der Halden méritait bien qu'un critique québécois s'y arrêtât. L'auteur des Études de littérature canadienne-française publiées en 1904 et des Nouvelles Études de littérature canadienne-francaise parues, comme le volume précédent à Paris chez F. R. de Rudeval en 1907, a, pendant dix ans, consacré tous ses moments libres à connaître et faire connaître cette littérature française d'outre-mer. Familier d'Hector Fabre et de l'abbé Casgrain, il correspond avec la plupart des écrivains canadiens. Halden sera directeur d'une collection canadienne chez F. R. de Rudeval où sera publiée, entre autres, L'Âme solitaire d'Albert Lozeau. Même si son désir de faire connaître les auteurs canadiens l'amène parfois à présenter une vision qui peut paraître excessivement positive de leurs œuvres, il faut remarquer qu'il s'agit, avant même Les Essais sur la littérature canadienne de Camille Roy, de la première tentative de présentation d'un corpus critique relativement complet et solidement documenté.

Marie-Andrée Beaudet met en évidence les nombreux points de convergence chez les deux polémistes: Fournier évoluera, tout en maintenant son ardeur critique face à l'état de la langue, vers une reconnaissance de plus en plus explicite de la naissance d'une littérature canadienne, allant même jusqu'à préparer une Antbologie des poètes canadiens! Halden, à la suite de Fournier et d'Arthur Buies, intègrera dans ses articles et dans les Nouvelles Études des préoccupations sur l'état de la langue française au Canada et son avenir incertain. Măis, note l'auteure avec raison, cette querelle

a agi sur le champ littéraire québécois a la façon d'un révélateur. Elle a obligé les uns et les autres écrivains et critiques, à prendre parti tout autant sur l'état présent de la langue et de la - littérature = que sur les critères de définition de l'ouuvre littéraire et du littéraire national. (p. 85)

Le programme de nationalisation littéraire dans lequel Camille Roy engage la littérature en ce début du $\mathrm{XX}^{\circ}$ siècle est aussi intrinsèquement lié à l'évolution du champ linguistique. C'est dans le Bulletin de la Société du Parler français que paraissent ses premières études sur l'histoire de la littérature canadienne; pour le critique l'obstacle majeur 
à l'épanouissement littéraire au Québec est linguistique. Mais on connaît la suite: épousant .l'approche défensive* de la Société du parler français au Canada, Camille Roy entreprend de distinguer la langue des campagnes, pure, héritière de celle du Grand Siècle, et la langue des villes, corrompue et anglicisée. Le choix entre les deux est vite fait et l'on engage les écrivains à emprunter la langue rurale pour créer une littérature nationale, laissant à la France la perfection d'une grande littérature. Roy assigne à notre littérature, menacée par les déficiences linguistiques, des objectifs infiniment plus modestes, qui en font un accommodement local, un pâle reflet de la seule grande littérature, la littérature française!

Dès lors les deux grandes avenues qui s'ouvrent aux littérateurs québécois sont parfaitement tracées. Ou bien suivre les mots d'ordre patoisants et archaïsants de la Société du parler français et d'Adjutor Rivard dont l'objectif était de produire un *dictionnaire" du français canadien *qui montrerait que notre langue est bien celle des ancêtres. (p. 119) et faire une littérature d'archives et de folklore dans la veine de la littérature régionaliste en France, ou bien tenter de sauver la langue d'ici, s'inscrire dans le sillon de l'École littéraire de Montréal et, comme Émile Nelligan, expérimenter la modernité. Si leurs effets sont esthétiques et éthiques, les choix se manifestent avant tout au niveau de la langue, comme s'il n'y avait jamais d'autre choix littéraire que celui-là.

L'essai de Marie-Andrée Beaudet est éminemment rafraîchissant, mettant à profit les études parues ou inédites (à quand la publication de la remarquable thèse d'Annette Hayward sur la querelle du régionalisme?) et apportant dans l'étude des rapports Fournier/ab der Halden des pièces nouvelles mais surtout un éclairage complètement renouvelé. L'essayiste met bien en évidence et établit plutôt deux fois qu'une le déterminisme absolu de la situation linguistique et des réactions qu'elle suscite sur les choix littéraires au Québec. L'ouvrage échappe à la sécheresse de la démonstration linéaire d'une thèse trop prévisible; rempli d'informations nouvelles, un peu comme le Xavier Marmier de Jean Ménard (1967), il ouvre tout au long de nombreuses perspectives à la réflexion critique et à la poursuite de recherches ultérieures. L'auteur a très bien compris le rôle des interrelations entre les discours critiques français et québécois: le point de vue sur la langue et la littérature c'est aussi le rapport à la France. En ce sens on peut peut-être regretter qu'elle n'ait pas poussé cette hypothèse jusqu'à sa limite et posé la France comme véritable horizon d'attente critique de presque toute cette littérature. La nationalisation terroiriste est bien une tentative de défrancisation. Mais les bons essais critiques ne sont-ils pas justement ceux que l'on a envie de poursuivre? 
1. Marie-Andrée Beaudet, Langue et Littérature au Québec, 1895-1914. L'impact de la situation linguistique sur la formation du cbamp littéraire, Montréal, L'Hexagone, coll. - Essais litteraires •, 1992, 221 p. 